\title{
ERRATUM: "HIGH-RESOLUTION IR ABSORPTION SPECTROSCOPY OF POLYCYCLIC AROMATIC HYDROCARBONS: THE REALM OF ANHARMONICITY” (2015, ApJ, 814, 23)
}

\author{
Elena Maltseva ${ }^{1}$, Annemieke Petrignani ${ }^{2,3}$, Alessandra Candian ${ }^{2}$, Cameron J. Mackie $^{2}$, Xinchuan Huang $^{4}$, \\ Timothy J. LeE ${ }^{5}$, Alexander G. G. M. Tielens ${ }^{2}$, Jos Oomens $^{3}$, and Wybren Jan Buma ${ }^{1}$ \\ ${ }^{1}$ University of Amsterdam, Science Park 904, 1098 XH Amsterdam, The Netherlands; w.j.buma@uva.nl \\ ${ }^{2}$ Leiden Observatory, Niels Bohrweg 2, 2333 CA Leiden, The Netherlands; petrignani@strw.leidenuniv.nl \\ ${ }^{3}$ Radboud University, Toernooiveld 7, 6525 ED Nijmegen, The Netherlands \\ ${ }^{4}$ SETI Institute, 189 Bernardo Avenue, Suite 100, Mountain View, CA 94043, USA \\ ${ }^{5}$ NASA Ames Research Center, Moffett Field, CA 94035-1000, USA \\ Received 2016 February 4; accepted 2016 February 16; published 2016 March 21
}

In the original manuscript, the middle panel of Figure 3 is accidentally identical to the middle panel of Figure 2. This erratum shows Figure 3 with the correct middle panel. Table 1 is unaffected, since it reports only experimental data. This change does not affect the scientific results of the paper.

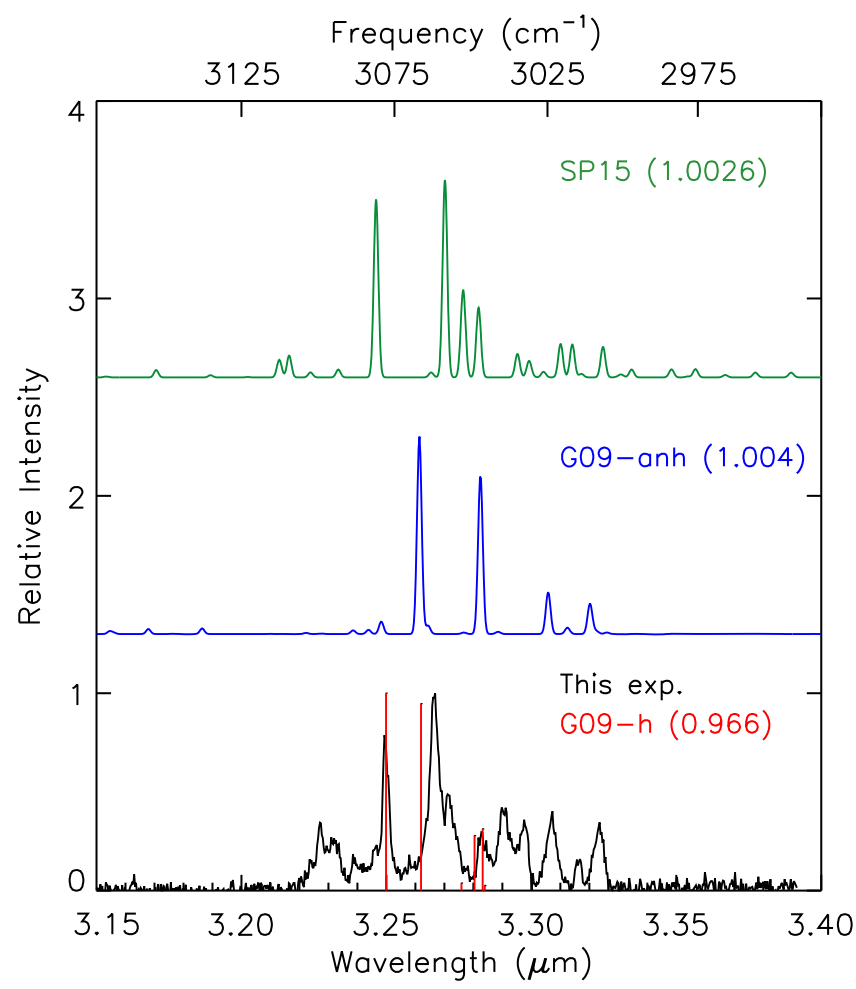

Figure 3. Absorption spectra of tetracene as predicted with (a) SP15, (b) G09-anh, and (c) G09-h together with the measured spectrum. 